(C) [2008] IEEE. Reprinted, with permission, from [Hoang, D.B.; Lawrence, E.; Ahmad, N.F.; Balasubramanian, V.; Homer, C.; Foureur, M.; Leap, N., Assistive care loop with electronic maternity records, Pe-health Networking, Applications and Services, 2008. HealthCom 2008. 10th International Conference on 7-9 July 2008]. This material is posted here with permission of the IEEE. Such ermission of the IEEE does not in any way imply IEEE endorsement of any of the University of Technology, Sydney's products or services. Internal or personal use of this material is permitted. However, permission to reprint/republish this material for advertising or promotional purposes or for creating new collective works for resale or redistribution must be obtained from the IEEE by writing to pubspermissions@ieee.org. By choosing to view this document, you agree to all provisions of the copyright laws protecting it 


\section{Assistive Care Loop with Electronic Maternity Records}

\author{
D. B. Hoang, E. Lawrence, N. F. Ahmad, V. \\ Balasubramanian \\ iNEXT- Centre for Innovation in IT Services and \\ Applications \\ University of Technology, Sydney, Australia \\ \{dhoang, elaine, nfahmad, vsubru\}@it.uts.edu.au
}

\author{
C. Homer, M. Foureur, N. Leap \\ Centre for Midwifery, Child and Family Health \\ University of Technology, Sydney Australia \\ \{Caroline.Homer, Maralyn.Foureur, Nicky.Leap $\} @$ uts.edu.au
}

\begin{abstract}
Surprisingly women-held pregnancy health records (paper based) are still predominantly used in most hospitals in Australia. These records are not standardized as each hospital or state has a slightly different version. Early efforts have been made to standardize pregnancy records and make them available electronically. Electronic record systems do not allow dynamic interaction between users and they are not accessible when users are mobile. This paper describes an Assistive Maternity Care (AMC) system that addresses a number of important issues: 1) transforming a women-held paper-based record for pregnancy care into an Electronic Maternity Record (EMR); 2) investigating mechanisms to make the record active; 3 ) creating a system whereby details of the pregnant women and their carers can be recorded, updated over wired and wireless networks; and 4) creating a pregnancy care loop over which midwives and doctors and pregnant women under their care can communicate effectively anywhere, anytime for the duration of pregnancy.

Keywords-Electronic Maternity Record; Assistive Care Loop; Active Database; Smartphone;Health Monitoring
\end{abstract}

\section{INTRODUCTION}

Woman-held paper-based health records have been demonstrated to be an effective method of record keeping in pregnancy. They allow pregnant women (users) more choice and control over their care during pregnancy and labour. Women (and their partners) can be involved in their own maternity care via such woman-focused records which can include personalised content, such as a plan for their labour and birth and designated areas where women can write questions. Currently, pregnant women only have a small version of their full health record to carry. Giving women their full paper-based record, known as the 'woman-held' record, means they can see their own results and follow their progress more closely than if they had a smaller version. Women who have access to their full record have reported feeling more informed about their pregnancy with higher levels of emotional well-being than those who do not [1].

The paper-based record in pregnancy has a number of disadvantages including poor use of space, fragility, the single user limitation at any one time, the ease with which records can be misplaced or lost, and the effort required in searching for information either in large single records, or from collections of records. Some efforts have been made in establishing an Electronic Maternity Record (EMR); however this remains limited to having the electronic record based in the hospital on desktops computers and accessible only by the midwives and doctors providing care [2, 3] Practitioners outside the hospital (e.g. general practitioners, community midwives) generally do not have access to the electronic information unless they are wired into the hospital network. The current electronic records are passive systems primarily used to collect data to produce annual statistics about birth outcomes. Pregnant women are passive and do not have a means to interact with the electronic system or even to see the data easily.

In maternity care, women are now expecting access to more information in order to make decisions about their care. In addition, maternity care is increasingly being based in the community away from the hospital setting. Mobility, in the form of smart phones or personal digital assistants (PDAs), is now required to ensure communication and health record keeping are effective and useful. Both influences are driving the development of this research.

This paper aims to addresses the two challenging research issues: active EMRs and mobility support, in order to provide a solution that improves the quality and safety of pregnancy care.

The specific objectives of the research were:

- to develop an electronic maternity record (EMR)

- to develop a mobile pregnancy (assistive) care loop to the EMR.

- to use the EMR to enhance communication between women and members of multidisciplinary teams who deliver maternity care.

\section{ISSUES IN DESIGNING THE ASSISTIVE CARE LOOP}

Our aim was to design an EMR, develop an architecture, and implement the assistive care loop that supports active interaction and user mobility. In this section, the design choices for building such an Assistive Maternity Care system are explored.

Transforming the paper women-held record to an electronic maternity record: To develop an EMR system from a paperbased record, we need to study and provide a 
comprehensive mapping between the two systems. While a paper-based record may have a flexible information structure, mapping it to an EMR presents challenges and requires careful consideration, especially with a mobile device which is limited in its display area. Meaningful presentation of information requires a major design which involves technology as well as user perception and usability.

For a smart phone or PDA, the issue of the display of information is critical because of the limitations of the device. Sometimes a coherent group of information may not be able to be grouped and placed together on one small screen and may be scattered over several screens or may have to be scrolled further down. Any rearrangement of displayed information must be acceptable to the users [4]. For desktop display, the issue is not as critical as the screen size is more or less adequate to accommodate what is on the paper. The issue is more about optimizing the content on the screen and navigation facility to make it easier for the user to operate and still have a familiar feeling and control over the displayed information.

During this development process, constant consultation with care providers (midwives) has occurred to ensure the outcome is user-friendly and has utility in the clinical setting.

Mobility: To be mobile, a user/device has to be able to be connected to the system to access the application data and interact with the provided services and other users. The Internet, wireless networks and mobile networks are essential for users to access the system anywhere anytime. The server and device have to be connected to the Internet and the application must be hosted by an Internet service provider for its services to be available to the users.

A user can access to the system and the application as long as he or she can connect to the Internet. The user can connect to the Internet through a wired connection through an Ethernet LAN or a high-speed modem (cable or ADSL) if the user is within reach of such a connection. If the user is mobile, he or she can wirelessly connect to the Internet through a wireless router or access point, or a wireless broadband network if he or she is within their reach. If the user is within the reach of a base station of a $3 G$ or GPRS mobile network, he or she can move freely about geographically and still connect to these wireless wide area networks. In some instances, the PDA can be directly connected to the server using its Bluetooth communication or its infrared communication ability [5]. In our setup we use a direct WiFi connection to the server in an ad hoc mode for development and then use the $3 \mathrm{G}$ network for testing access in wide area networks.

Security: It is important that the system and its users are protected with security mechanisms. These mechanisms are required for both data transfer over the communication network but also for accessing the application and its data within the system.

For secured data transfer, we rely on the security mechanisms of the $3 \mathrm{G} / \mathrm{GPRS}, \mathrm{WiFi}$ and secured Internet service provider's networks.
For system access, each user of the system must be authenticated. For example, a midwife has to be authenticated before he or she can register to the system.

A role-based approach is used for authorization. Selective information and interfaces are accessed depending on the user's role. For example, the role of the midwife or doctor must be defined with proper access rights to ensure that the display is only relevant to the role and can only access the information of the pregnant women under his or her care. Similarly, a pregnant woman must be authenticated before she can access her own information. She cannot get access to other user's data. In particular, personal details of the woman are only displayed when an authentication test (password) is passed every time the details are requested to prevent unauthorized users from accessing personal details.

Another related issue is that a mobile device has high probability of being lost (even when it is being used). It is best that personal details are further protected. One solution is to keep as little information as possible within the device.

Activeness: Most systems allow users to get access to its contents upon request. Many useful systems are built with such a client/server paradigm. However, it is more effective if the system can react autonomously to changes in its environment or be responsive to conditions generated by users. Such system is active. We have focused on making the data record active. The choice is in determining and selecting relevant components of the record and making it active. If data supplied from the user satisfies certain condition, the system will trigger some actions and generate some response as defined by a set of rules.

Furthermore we define a set of protocols to allow active and effective communication and interaction between the midwife and the pregnant women through the system.

\section{DESIGN AND IMPLEMENTATION OF THE ASSISTIVE MATERNITY CARE SYSTEM}

A paper-based system for keeping the records of pregnancy information has been designed and implemented in one health service in Sydney. Our new system that we describe in this paper transforms the paper version into an electronic version. The EMR retains all the information contained in the paper-based system with future extension to include features that come about because of the advantages of EMR. Figure 1 illustrates our overall system. It functionality can be broken down into three main components: the health server system, the client system, and the communications infrastructure supporting the applications. The health server system consists of an application and its EMR records, an application container, a web server and a database server. The client system consists of PDAs and their users. The infrastructure consists of wired and wireless mobile networks.

The health server system:

The application is where the module that contains all intelligent logic for processing users request by interacting with other components and making decisions. For example, 
it controls the access right of users, and processes user data to see if any action is needed, etc.

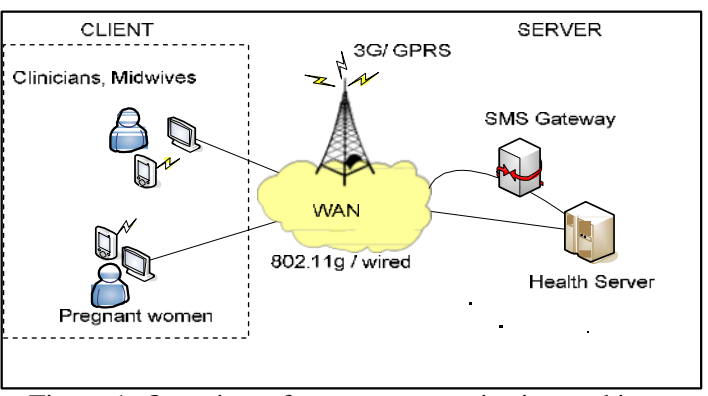

Figure 1: Overview of pregnancy monitoring architecture

The web server receives user requests and redirects them to the application through the application container. It also receives responses from the application and sends them back to the browser on the user's PDA.

The application container in this case is the Apache Tomcat which houses an environment for developing and executing the application.

The database server allows databases to be created and serves as a repository for user's information. It also contains the facilities for triggering simple actions such as sending reminder and notification via SMS or email under some prescribed conditions, for example, abnormal range of monitored parameters, reminder of visit date and re-test date, etc.

The client system.

The smart phone is capable of connecting to the Internet through various technologies. It can either connect to the Internet and then to the server system using $\mathrm{WiFi}$ or 3G/2.5GPRS mobile networks. Communication with the application is through the web browser.

A user can operate the smart phone to interact with other users and the system. Users can be pregnant women or clinicians which includes midwives or doctors. The interfaces of the system allow a registered clinician to access the system to inspect data records of pregnant women under his or her care. The system also provides interfaces for the clinician to provide appropriate advice or responses.

A pregnant woman can access through the PDA's interface, information concerning her pregnancy record or enter data to update her personal details. The woman can also submit queries concerning her pregnancy to clinicians.

Besides these data interfaces, a clinician and the pregnant woman can always interact through a phone conversation as necessary. In addition, clinicians can also discuss various conditions about pregnant women among themselves. If a smart phone or a connection to the system via smart phone is not available, users can interact with the application through an Internet-connected desktop computer.

The communication infrastructure
A $3 \mathrm{G}$ or GPRS mobile network is used to support wireless wide area connection between the server and the client systems. These networks support both voice and data communication within the coverage of the networks.

A client system can also connect to the server system if it is connected through WiFi networks. However, the coverage of WiFi is often limited to local areas and hot spots.

\section{The Assistive Maternity Care (AMC) System}

Our AMC system is based on an existing paper-based woman-held record that is being implemented in a New South Wales (NSW) Area Health Service. The system can be accessed through a web browser at a desktop or on a PDA. In this first phase of the pilot, we designed and developed several modules which include Woman and Clinician Details, Pregnancy Details, Diagnostic Details, Visit Details, and Discussion Details and Admission Details [1]. We designed each module so that it is systematically mapped to the real procedure of pregnancy management in the NSW maternity unit.

In the paper version, each pregnant woman was given a booklet that records all details and information about her pregnancy. If the woman wants to discuss any concerns, she can write them down on the space provided in the booklet. Her clinician will respond to the written concerns when the woman visits the health service for the next regular checkup. It is clear that with the current paper-based system the interaction between women and clinicians is extremely limited. With the implementation of the EMR, the AMC system provides a platform for the pregnant woman to interact with her carer dynamically and conveniently. The woman does not have to wait until the next visit to discuss her concerns. She can enter and send her queries whenever she desires through the client interface and her clinician can view and respond to them as necessary. The system works asynchronously but on average the response time is much faster than the next visit.

In addition, the AMC system also can send reminder to the woman about certain incoming events. For instance, a reminder about regular visit date or about re-test date can be sent to the women automatically and in advance, based on the EMR record. Figure 2 illustrate the dynamic interaction between the women and the clinicians or midwives.

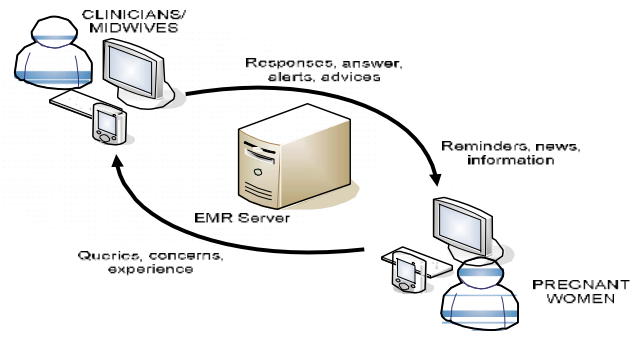

Figure 2: Assistive care loop between pregnant women and clinicians/midwives 


\section{The Active EMR}

An EMR provides a uniform set of necessary information about a pregnant woman and her pregnancy. This includes details about the woman's personal data, her current pregnancy, her pregnancy history, the result of the pregnancy diagnostic, and other information. An active EMR is a specially designed EMR in which a set of selected components of the EMR are made "active". These components are monitored continuously and when their values or combination of their values satisfy a predefined condition, an action will be initiated by the system. As a result, an alert or a response will be delivered to the woman and appropriate clinicians. Active EMR is achieved through the use of active database systems and intelligent activation schemes.

An active database system (ADS) is a database in which the operation is automatically executed when certain condition arises [6]. ADS extend passive database systems' functionality with active rules. Active rules allow ADS to monitor the stored data, to respond automatically to the events and to execute defined actions in timely manner. Active rules can be achieved through trigger and event functions.

In future models, it would be possible to monitor pregnant women with hypertension. The crucial parameters, which are the values of blood pressure, are monitored using triggers. Figure 3 illustrates how the monitoring is implemented using trigger. When pregnancy data is entered into Visit table, the trigger checks the systolic and the diastolic value. If the value of systolic is within the normal range, the trigger will not be executed. In contrast the trigger will insert the systolic values and details of woman in MonBP table. The same process applies to diastolic values. If it is not in normal range, the diastolic values and the correspond details of woman is forwarded to MonBP table.

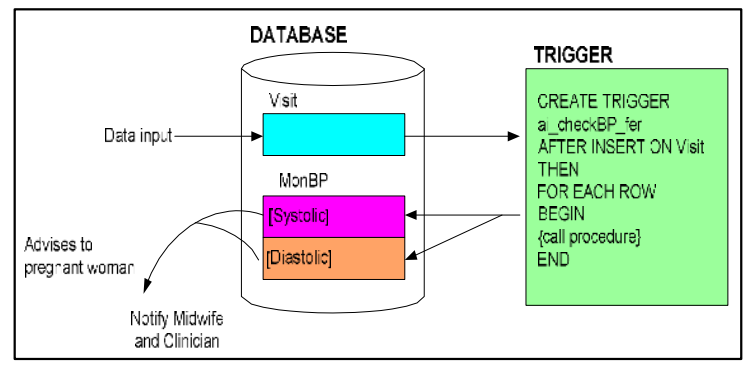

Figure 3: Example of trigger for active database

\section{RESULTS AND DISCUSSION}

The AMC system has been partially implemented. From the user's view point, the system has three interfaces: the administrator, the clinician, and the pregnant woman interfaces. These interfaces can be accessed from a desktop computer or a smartphone as long as it is connected to the Internet.
The administrator interface

The role of an administrator or a chief clinician in the context of this system includes the clinician's application approval and the termination of clinician's account. The administrative interface allows the administrator to display the details of the clinician who apply for registration before approving their access to the system. The module also supports termination function of the clinician access. This is necessary in a case of when the clinician is shifted to different hospital or location. In addition, the administrator can display list of registered clinicians and assigned the responsible clinician to each of the pregnant woman.

\section{The clinician interface}

The clinician interface is the main interface where its hold most of the functionality of the system. This interface allows a clinician to register as a midwife, who is looking after several pregnant women under their care. The registration requires an approval of an administrator who can authenticate the clinician and send an email with password to the registered clinician. Once registered, the clinician can display the EMR of a particular pregnant woman to review the progress of the pregnancy case.

As shown in Figure 5(d), link to each of the module that we previously mentioned is presented using dropdown menu. First, Pregnancy Details where this module contains the date of important occurrences such as last menstrual date, expected due date, gestation weeks, etc. Secondly, Result Details, which stored all diagnostic results taken by the woman. Next module is Visit Details (Figure 5(f)). This module displayed the regular checkup information. In each visit, the clinician and the woman discussed several topics which are listed in Discussion Details. The last link is Admission module where the data of admission to the hospital is recorded.

For each of the above module, the clinician can view the current and the previous maternity information of the woman. The clinician can add and update the maternity record of the particular woman during their regular checkup. Some of the screen shots of the EMR record appear below

The pregnant woman interface

This interface allows registered pregnant women to view and modify her personal details. The pregnant woman can access to a similar set of screens as seen by the physician. She can enter, and modify some relevant data as appropriate, however, she has limited access rights to those data field and screens. For example, she may not overwrite information and data entered by the clinician. The current and previous pregnancy information provides a means for the woman to acknowledge and keep her updates with her current condition. For our pilot study, the pregnant women under study will mainly access the AMC system through a desktop interface as shown in Figure 4.

In particular, for the case of high blood pressure (BP), whenever the BPs value entered to the EMR record falls within the abnormal range, the system automatically evaluates the data and sends an alert to the woman advising her to follow a follow-up procedure such as taking a BP test. 
If necessary, the clinician will be alerted and he or she will initiate a conversation with the woman for further advice and action.

This paper mainly describes a basic AMC system yet many features of the systems are not discussed in detail due to the space limit and some are still evolving and being developed.

Comments on the interfaces: Each of the interfaces has been designed after consultation with the end users including pregnant women, midwives, health workers, academics and a number of test users of differing ages. The best features of the paper-based EMR were incorporated into the new system and improvements such as drop down boxes (not available in paper based systems) were added. Of key importance was the necessity to ensure that the interface worked equally well on a laptop/desktop screen and a PDA. The small screen realestate on a PDA presented the following challenges:

1. ensuring that the user did not have to continually scroll to see the data

2.displaying the data in appropriate size chunks

Comments on accessibility: The system improves accessibility of the records to a number of persons. In the paper based system, the pregnant woman held the record and the caregivers only saw the record when she brought it in to the midwife or doctor during a visit. The EMR means that the record can be viewed by authorized persons via the internet when required. The pregnant woman has to ability to contact her caregivers electronically when necessary. Comments on security: The vexed issue of security has been addressed by the use of authentication and authorization. To be authenticated as a legitimate user of the system a user must have appropriate credentials such as a valid password. Authorization refers to the level of access a particular user has - so in our system a healthcare worker has access to certain medical tables to which a pregnant woman does not have access, for example. If the PDA is lost or stolen an unauthorized person is not able to access the PDA itself which is also protected by a password.

Comments on the cost: Most people today have a mobile phone so the use of the smart phone is not much of a problem. However, it is still relatively expensive to connect to the Internet through a Wireless Service Provider.

Comments on the integration with existing infrastructure: One particular feature is the design of the active EMR. This feature describes a particular case study as the process of selecting and making components of an EMR active is highly dependent on the particular application. However, techniques for transforming a passive EMR to an active one can still be generic and useful for all applications. Further protocols for dealing with these dynamic interactions are required.

\section{CONCLUSION}

The paper introduced the "active" concept in Maternity Record and deployed wired and wireless networking technologies to support an assistive care application. It transformed a paper form women-held's record to an EMR embedded with selective active components. It described a system that centers on a database of these EMRs and established a pregnancy care loop over which midwives and their carers can interact effectively anywhere, anytime. The system is being tested and the results will be summarized in a later report. We believe that the outcomes will open up several new areas of research: The EMR could be adapted and tailored to many task-specific applications that involve data collections and interactions between users. It could be extended to include sensors for health monitoring systems for monitoring others, for example, people who are aged, have chronic disease or after surgery. It could be extended to allow multidisciplinary collaboration concerning a person's health.

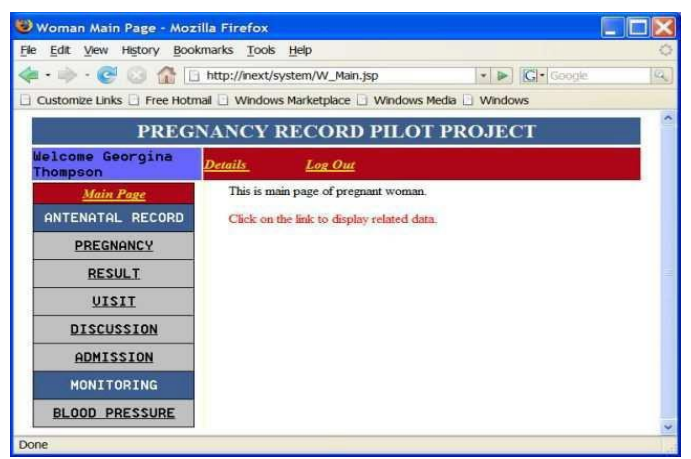

(a)

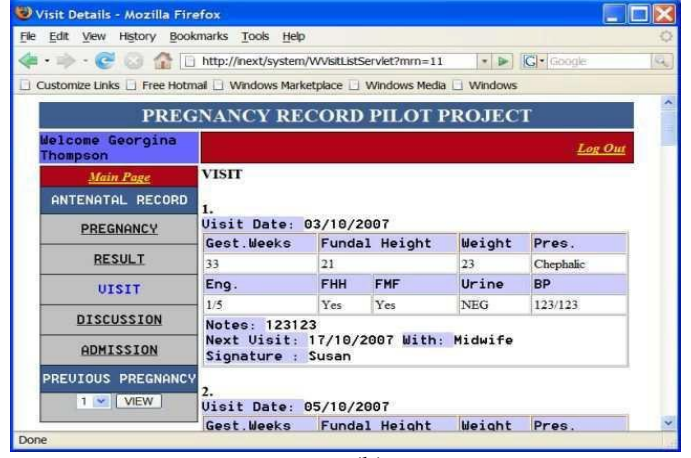

(b)

Figure 4. Desktop interface for woman: (a) list of options for viewing, (b) details of visits

\section{ACKNOWLEDGMENT}

This research was supported under Australian Research Council's Discovery Projects funding scheme (project number DP0774207) and UTS Challenge Grants (RM No: 2006001132)

\section{REFERENCES}

[1] C. Homer, G. Davis, and L. Everitt, "The Introduction of a WomenHeld Record into a Hospital Antenatal Clinic: The Bring Your Own 
Records Study," Aust NZ J. Obstet Gynaecology, vol. 39, pp. 54-57, 1999.

[2] Bedford H, Chalmers J. A, "New Vision for Maternity Records in Scotland:The Scottish Woman-Held Maternity Record (SWHMR) Project and the electronic Scottish Woman-Held Maternity Record (eSWHMR) Project." in Journal of the Institute of Health Record Information and Management, vol 44 (2), pp. 21-24, 2003

[3] Victorian Government Health Information, "Current Programs/Initiatives: Victorian Maternity Record (VMR)", Maternity Care in Victoria, http://www.health.vic.gov.au/maternitycare/progs.htm
[4] S. Uppu, D. B. Hoang., and T. Hintz, "A Mobile Hand Held Computing System for Outpatient Workflow in Hospital Environment," in The 63rd IEEE Vehicular Technology Conference Melbourne, Australia: IEEE Press, 2006.

[5] D. B. Hoang and A. Simmonds, "Chapter 10. Data Communication and Network Infrastructure," in Biomedical Information technology, D. Feng, Ed.: Academic Press, 2007, pp. 229-248.

[6] N. W. Paton and O. Diaz, "Active Database Systems," ACM Computing Surveys, vol. 31, pp. 63-103, March 1999.

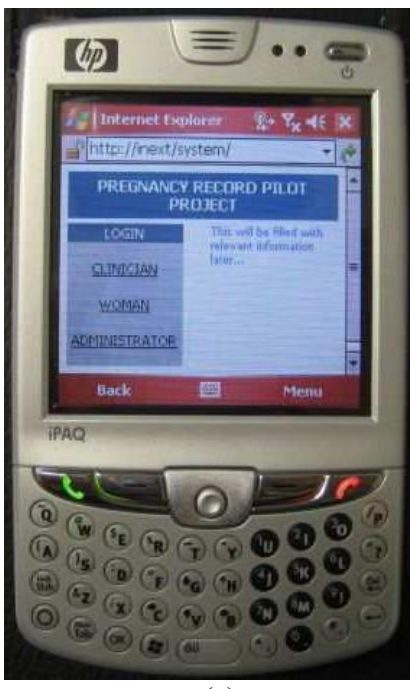

(a)

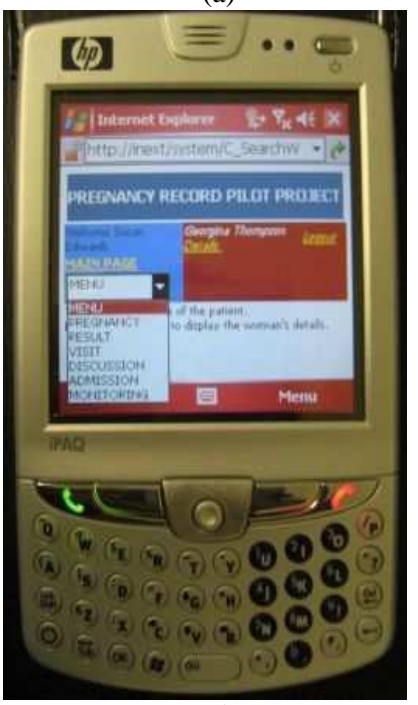

(d)

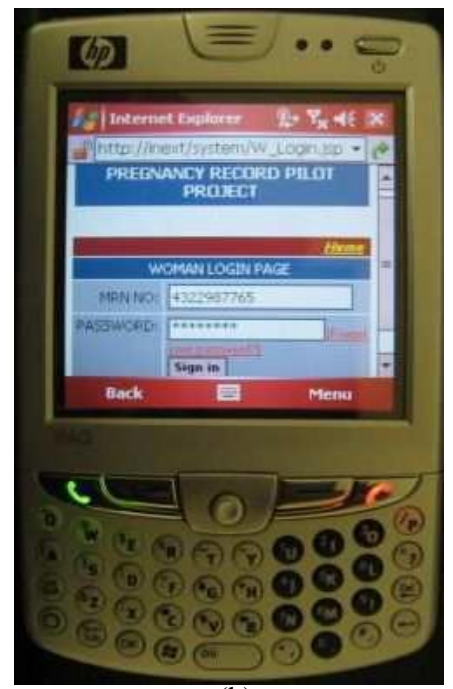

(b)

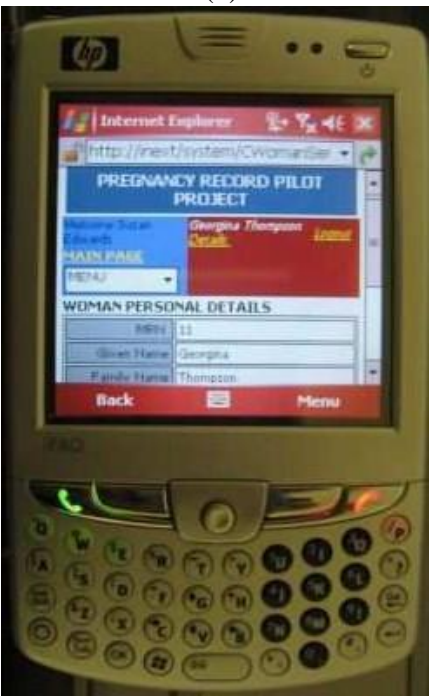

(e)

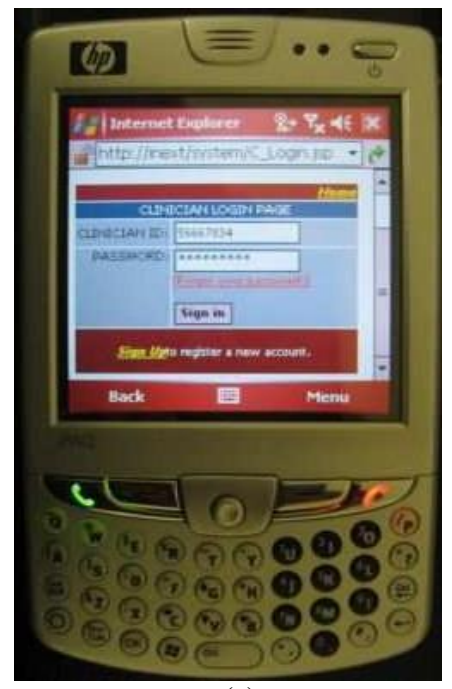

(c)

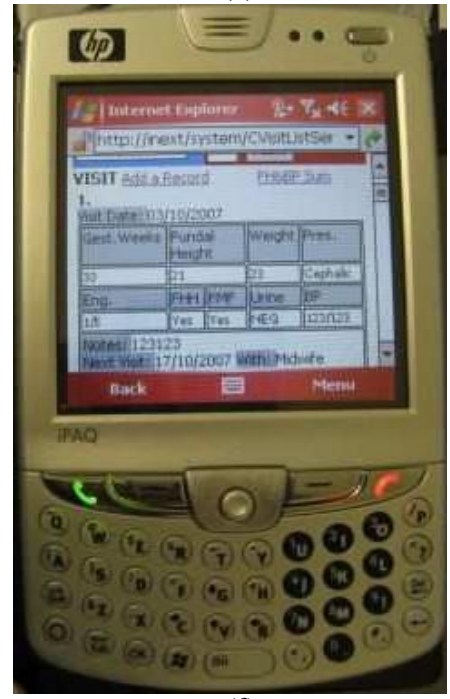

(f)

Figure 5. PDA Interfaces: (a) AMC homepage, (b) clinician login, (c) woman login, (d) drop-down menu for viewing selection, (e) woman's details, (f) details of visits 